\title{
Upcoming Meetings Related to Alzheimer's Disease*
}

\section{SEPTEMBER 2004}

Conference:

Date:

Location:

Contact:

Conference:

Date:

Location:

Contact:

Conference:

Date:

Location:

Contact:

\section{OCTOBER 2004}

Conference:

Date:

Location:

Contact: 8th Congress of the European Federation of Neurological Societies 4-7 September 2004

Palais des Congrès, Paris, France

headoffice@efns.org

Phone: +4318890503

Fax: +431889050313

http://www.kenes.com/efns2004/index.html

\section{Biology of Aging}

12-17 September 2004

Centre Paul Langevin, Aussois, France

Julie Andersen - JANDERSEN@BUCKINSTITUTE.ORG

Buck Institute for Age Research, 8001 Redwood Boulevard, Novato, California, 94945, USA

http://www.grc.uri.edu/programs/2004/aging.htm

II International Congress on Neurodegenerative

20-24 September 2004

Rio de Janeiro

Congress Secretariat -icn@congrex.com.br

Phone: +55 2139742000

Fax: +55212507 1492

http://www.neuroregeneration2004.med.br

Symposium on Nutrition and Alzheimer's Disease

1-2 October 2004

New Takanawa Prince Hotel, Tokyo, Japan

Akira Ueki - office@ianajapan.com

Fax: +81352165552

Jichi Medical School, Omiya Medical Center, 1-847 Amanuma-cho,Omiya-ku, Saitama, City 330-8503, Japan

http://www.ianajapan.com/eng.html

Conference: $\quad$ 129th Annual Meeting of the American Neurological Association

Date: $\quad 3-6$ October 2004

\footnotetext{
*Derived from listings at Alzheimer Research Forum (http://www.alzforum.org).
} 
Location:

Contact:

Conference:

Date:

Contact:

Conference:

Date:

Location:

Contact:

Conference:

Date:

Location:

Contact:

Conference:

Date:

Location:

Contact:

Conference:

Date:

Location:

Contact:
Location:

Toronto, Ontario, Canada

American Neurological Association - lorijanderson@msn.com

Phone: +1952 5456284

5841 Cedar Lake Road, Suite \#204, Minneapolis , Minnesota, 55416, USA

The Human Brain: Modelling and Remodelling

5-9 October 2004

IRCCS Foundazione Santa Lucia, Rome, Italy

secretariat@thehumanbrain.org

Phone: +39065015636

Fax: +39065015636

http://www.thehumanbrain.org/

Molecular Genetics of Aging

6-10 October 2004

Cold Spring Harbor Laboratory, Cold Spring Harbor, NY, USA

Cold Spring Harbor Laboratory Meetings \& Courses Programs Office meetings@cshl.edu

Phone: +1 5163678346

Fax: +1 5163678845

1 Bungtown Road, Cold Spring Harbor, New York, 11724-2213, USA http://meetings.cshl.org/2004/2004aging.htm

20th International Conference of Alzheimer's Disease International 15-17 October 2004

Kyoto International Conference Hall, Kyoto City, Japan

Yoshio Miyake MD - adiconference@alzheimer.or.jp

Phone: +81 758236544

Fax: +81758236545

http://www2f.biglobe.ne.jp/\%7Eboke/boke2-e.htm

34th Annual Meeting of the Society for Neuroscience

23-28 October 2004

San Diego, CA, USA

Society for Neuroscience

Phone: +1 2024626688

http://info@sfn.org

Mental Dysfunctions in Parkinson's Disease

24-27 October 2004

Salzburg, Austria

PDment2004@kenes.com

Phone: +4122908 0488

Fax: +4122732 2850

17 Rue du Cendrier, P.O. Box 1726 CH-1211, Geneva 1, Switzerland http://www.kenes.com/pdment2004/index.html

\section{DECEMBER 2004}

Conference:

Drug Discovery in Neurodegenerative Diseases 
Date:

Location:

Contact:

Conference:

Date:

Location:

Contact:

\section{MARCH 2005}

Conference:

Date:

Location:

Contact:

\section{APRIL 2005}

Conference:

Date:

Location:

Contact:

\section{MAY 2005}

Conference:

Date:

Location:

Contact:

JUNE 2005

Conference:

Date:

Location:

Contact:
2-5 December 2004

Cold Spring Harbor Laboratory, Cold Spring Harbor, NY, USA

Cold Spring Harbor Laboratory Meetings \& Courses Programs Office

meetings@cshl.edu

Phone: +1 5163678346

Fax: +15163678845

PO Box 100, 1 Bungtown Road, Cold Spring Harbor, New York, 11724-2213, USA

http://meetings.cshl.org/2004/2004degen.htm

Alzheimer's Disease: From Molecular Mechanisms to Drug Discovery

11-17 December 2004

Cancun, Mexico

Nico Stanculescu - meetings@worldeventsforum.com

Phone: +1 7737848134

Fax: +1 2085755453

5030 N Marine Drive, Suite 2608, Chicago, Illinois, 60640, USA

http://www.worldeventsforum.com/ad/index.html

7th International Conference AD/PD 2005

9-14 March 2005

Sorrento, Italy

adpd@kenes.com

Phone: +41 229080488

Fax: +4122732 2850

Kenes International, 17 Rue du cendrier, Geneva, Switzerland

American Academy of Neurology 57th Annual Meeting

9-16 April 2005

Miami, FL, USA

web@aan.com

Phone: +1 6516951940

American Academy of Neurology, 1080 Montreal Avenue, St. Paul, Minnesota, 55116, USA

6th World Congress on Brain Injury

1-4 May 2005

Melbourne, Australia

braininjury@icms.com.au

16th International Congress on Parkinson's Disease and Allied Disorders

5-9 June 2005

Berlin, Germany

berlin@cpo-hanser.de

Phone: +49303056690

Paulsborner Strasse 44, D-14193, Berlin, Germany 
Conference:

Date:

Location:

Contact:
XVIII World Congress of Gerontology: New Perspective

26-30 June 2005

SãPaulo, Brazil

Norton Sayeg - nortonsa@uol.com.br

Phone: +55 1130816247

Rua Augusta 2709, 64 São Paulo, São Paulo, Brazil

http://www.rio.rj.gov.br/

\section{SEPTEMBER 2005}

Conference:

Date:

Location:

Contact:

Conference:

Date:

Location:

Contact:

\section{NOVEMBER 2005}

Conference:

Date:

Location:

Contact:

Conference:

Date:

Location:

Contact:

Conference:

Date:

Location:

Contact: 9th Congress of the European Federation of Neurological Societies

17-20 September 2005

Congress Centre OMMA of the Megaron-Athens Concert Hall, Athens, Greece

efns05@kenes.com

Phone: +4122908 0488

Fax: +4122732 2850

17 Rue du Cendrier, PO Box

1726, Geneva, CH-1211, Switzerland

http://www.kenes.com/efns2005/

130th Annual Meeting of the American Neurological Association

26-29 September 2005

San Diego, CA, USA

lorijanderson@msn.com

5841 Cedar Lake Road, Suite \#204, Minneapolis, Minnesota, 55416, USA

World Congress of Neurology 2005

5-13 November 2005

Sydney, Australia

The Neurological Society of Thailand Division of Neurology, Department of Medicine info@thaineurology.org

Phone: +6622011386

Ramatibodi Hospital Mahidol University, Phayathai Road, Bangkok, 10400, Thailand

18th World Congress on Neurology

5-13 November 2005

Sydney Convention \& Exhibition Centre, Sydney, Australia

conference@wcn2005.com

Phone: +61292411478

Fax: +61292513552

GPO Box 2609, Sydney, NSW 2001

http://www.wen2005.com

35th Annual Meeting of the Society for Neuroscience

12-16 November 2005

Washington, DC, USA

info@sfn.org

Phone: +1 2024626688

11 Dupont Circle, N.W., Suite 500, Washington, DC, 20036, USA 